Sharif University of Technology
Scientia Iranica
SCIENTIA
IRAN I CA

\title{
The numerical solution to the Bagley-Torvik equation by exponential integrators
}

\author{
S. Esmaeili* \\ Department of Applied Mathematics, University of Kurdistan, P.O. Box 416, Sanandaj, Iran.
}

Received 30 May 2016; received in revised form 9 July 2016; accepted 29 October 2016

\author{
KEYWORDS \\ Fractional derivatives; \\ Fractional differential \\ equations; \\ Bagley-Torvik \\ equation; \\ Mittag-Leffler \\ function; \\ Exponential \\ integrators.
}

\begin{abstract}
This paper presents a family of computational schemes for the solution to the Bagley-Torvik equation. The schemes are based on the reformulation of the original problem into a system of fractional differential equations of order $1 / 2$. Then, suitable exponential integrators are devised to solve the resulting system accurately. The attainable order of convergence of exponential integrators for solving the fractional problem is studied. Theoretical findings are validated by means of some numerical examples. The advantages of the proposed method are illustrated by comparing several existing methods.
\end{abstract}

(C) 2017 Sharif University of Technology. All rights reserved.

\section{Introduction}

Studying fractional calculus is an old topic in mathematical analysis, which goes back to Leibniz (1695) and Euler (1730) (cf. [1, Section 1.1]). Even though the topic of fractional calculus has a long history, it, however, has drawn the attention of mathematical communities and of specialized conferences only in the last 40 years. Due to new developments in the analysis and understanding of many complex systems in engineering and science fields, it has been observed that several phenomena are more realistically and accurately described by differential equations of fractional order. The well-known anomalous diffusion process is one of the most typical examples. Other applications of fractional calculus are linear viscoelasticity, electrical circuits, nuclear reactor dynamic, electrochemistry or image processing (cf. [2-8]).

The operator of fractional derivative is more

\footnotetext{
*. Tel./Fax: +98 8733624133

E-mail address: sh.esmaeili@uok.ac.ir
}

complicated than the classical one. As a result, its calculation is also more difficult than the integer order case. Unlike integer order derivatives, which are local operators, the presence of integral in non-integer order derivatives makes the problem global. For this reason, fractional derivatives are powerful tools to describe processes with memory effects and long-range dispersions. On the other hand, the presence of a significantly persistent memory, with respect to the integer order case, adds more complexity to the numerical treatment of the related differential problems, especially for longtime integration.

Since few of the Fractional Differential Equations (FDEs) encountered in practice can be solved explicitly, it is necessary to employ numerical techniques to find the approximate solution (cf. $[2,6,9]$ ).

One of the numerical methods that has become increasingly applicable in recent years is exponential integrators (cf. [10-12]). Exponential Integrators (EIs) are a class of powerful methods specifically designed for solving semi-linear ordinary differential equations. Basically, the linear term is separated and solved by a matrix exponential and a time-stepping technique is applied to the nonlinear term (cf. [13]). Even though 
EIs were introduced in the early 1960s, they have received little attention in the past due to the difficulty in evaluating functions with matrix arguments. In recent years, the spread of efficient methods for performing this task has renewed interests in EIs (cf. [14]).

The seminal paper of Torvik and Bagley (cf. [15]) first proposed the Bagley-Torvik equation. It has an outstanding role of being a model of motion of a rigid plate immersed in a Newtonian fluid. The stability and asymptotic properties of the homogeneous BagleyTorvik equation are discussed in [16]. Numerical schemes for the Bagley-Torvik equation have been developed in the past ten to fifteen years and it has been studied in numerous papers. In [17], the BagleyTorvik equation is solved by first reducing this equation to a system of FDEs of order 1/2, and then fractional linear multistep methods and a predictor-corrector method of Adams type are used. Lately, the BagleyTorvik equation has been numerically solved by hybrid functions approximations [18], a well-posed Chebyshev Tau method [19], the Haar wavelet operational matrix [20], and the Bessel collocation method [21]. For further analytical and numerical methods, readers are referred to [22-28].

The main goal of this paper is to design efficient numerical schemes for numerical solution to the Bagley-Torvik equation. To do this, the BagleyTorvik equation is first transformed into a system of linear FDEs of commensurate order $1 / 2$, and then by means of the variation-of-constants formula, the exact solution to this problem is obtained. This exact solution does not necessarily provide the best way to compute the solution numerically. Indeed, it typically contains two difficulties: evaluating the Mittag-Leffler functions with matrix argument and performing the integration analytically. Under certain circumstances, the matrix coefficient has eigenvalue de-composition and the first difficulty can be removed. Since most of such integrals cannot be evaluated explicitly, EIs can be used to overcome the second difficulty. The resulting method provides promising results. Compared to classical approaches, the polynomial approximation is not applied to the whole vector field or to the solution of the FDEs, which is a source of inaccuracy in fractional order problems, but just to the external source term that is usually sufficiently smooth, and hence can be approximated in a satisfactory way by means of polynomials.

The structure of the paper is as follows. In the next section, a brief summary of fractional operators and related special functions is given. In Section 3, the derivation of the Bagley-Torvik equation is first briefly recalled, and then this equation as a system of FDEs of order $1 / 2$ is reformulated. In Section 4, EIs for fractional problem are devised and a description of the proposed numerical scheme is provided. In Section 5, the convergence properties are studied. Finally, the numerical results demonstrating the efficiency of the proposed method are presented in Section 6 .

\section{Mathematical preliminaries}

In this section, some of the main ideas about basic concepts, which will be used later in this paper, are sketched.

\subsection{Fractional derivatives}

There are many definitions of fractional derivatives (cf. $[2,6])$; the two most commonly used definitions are those referred to as Riemann-Liouville and Caputo. Their definitions are presented here.

Let $\alpha \geq 0$ and $m=\lceil\alpha\rceil$ be the smallest integer, such that $m>\alpha$. In mathematical treatises on FDEs, the Riemann-Liouville approach to the notion of the fractional derivative of order $\alpha$ and with the starting point at $t=0$ is normally used:

$$
{ }_{0} D_{t}^{\alpha} y(t):=D^{m} J_{0}^{m-\alpha} y(t), \quad t>0
$$

where $D^{m}$ denotes the classical differential operator of integer order $m$ and for locally integrable function $y$ :

$$
J_{0}^{\nu} y(t):=\frac{1}{\Gamma(\nu)} \int_{0}^{t}(t-s)^{\nu-1} y(s) \mathrm{d} s,
$$

is the Riemann-Liouville fractional integral of order $\nu$ $(\nu>0)$.

It is well known that differential operator $D^{\alpha}$ has an $m$-dimensional kernel; therefore, there is certainly a need to specify $m$ initial conditions in order to obtain a unique solution to the straightforward form of a FDE:

$$
D^{\alpha} y(t)=f(t, y(t)),
$$

with some given function $f$ (cf. [29]). As to the initial value problem for FDEs with the fractional derivatives in the Riemann-Liouville sense, there are some troubles with the initial conditions (cf. $[2,30]$ ). Namely, these initial conditions are in the form of:

$$
\begin{aligned}
& { }_{0} D_{t}^{\alpha-k} y(0)=b_{k}, \quad k=1, \cdots, m-1, \\
& \lim _{t \rightarrow 0+} J_{0}^{m-\alpha} y(t)=b_{m},
\end{aligned}
$$

with given values, $b_{k}$. However, it is not clear what the physical meaning of a fractional derivative of $y$ is, and hence it is also not clear how such a quantity can be measured.

A certain solution to this conflict was introduced by Caputo and later adopted by Caputo and Mainardi in the framework of the theory of linear viscoelasticity (cf. $[2,5,9])$. Caputo's definition can be written as follows:

$$
D_{*}^{\alpha} y(t)=J_{0}^{m-\alpha} D^{m} y(t), \quad t>0 .
$$


However, the situation is different when dealing with Caputo derivatives. These conditions have the form of:

$$
D^{k} y(0)=c_{k}, \quad k=0,1, \cdots, m-1,
$$

where real numbers $c_{k}$ are assumed to be given.

As a consequence of definitions (1) and (2) at case $\alpha \in \mathbb{N}$, these fractional derivatives coincide with the classical integer order derivatives (cf. [2,9]). It is well known that the fractional derivatives of RiemannLiouville and Caputo type are closely linked by the following relationship:

$$
D_{*}^{\alpha} y(t)={ }_{0} D_{t}^{\alpha}\left[y(t)-T_{m-1}(t)\right],
$$

where $T_{m-1}$ denotes the Taylor polynomial of degree $m-1$ for function $y$, centered at $t=0$.

In order to approximate fractional derivatives, a number of methods have been proposed (cf., e.g., $[6,31])$.

\subsection{Generalized Mittag-Leffler functions}

The Mittag-Leffler (ML) function plays a fundamental role in fractional calculus. The ML function with two parameters $\alpha, \beta \in \mathbb{C}$ is defined by means of the series expansion:

$$
E_{\alpha, \beta}(z)=\sum_{k=0}^{\infty} \frac{z^{k}}{\Gamma(\alpha k+\beta)}, \quad \Re(\alpha)>0 .
$$

The relevance of this function appears nowadays in certain fractional relaxation and diffusion phenomena [32]. Indeed, the eigenfunction of a FDE, i.e. the solution to the linear test equation $D_{*}^{\alpha} y(t)=\lambda y(t)$, can be expressed in terms of the ML function as $y(t)=$ $E_{\alpha, 1}\left(\lambda t^{\alpha}\right) y_{0}$, where $y(0)=y_{0}$ is the initial value.

Recent developments in the solution of FDEs involve a generalization of ML functions, namely:

$$
e_{\alpha, \beta}(t ; \lambda)=t^{\beta-1} E_{\alpha, \beta}\left(t^{\alpha} \lambda\right), \quad t>0, \quad \lambda \in \mathbb{C},
$$

which plays an important role in the analytical solution of FDEs. In some situations, it is of practical value to scale time variable, $t$, according to the equivalence:

$$
e_{\alpha, \beta}(t ; \lambda)=h^{\beta-1} e_{\alpha, \beta}\left(t / h ; h^{\alpha} \lambda\right), \quad h>0,
$$

whose proof can be easily derived by using the series representation of $\mathrm{ML}$ function, $E_{\alpha, \beta}$. The Laplace transform of $e_{\alpha, \beta}(t ; \lambda)$ is (cf. [2,(1.80) for $\left.k=0\right]$ ):

$$
\mathcal{E}_{\alpha, \beta}(s ; \lambda)=\frac{s^{\alpha-\beta}}{s^{\alpha}-\lambda}, \quad \Re(s)>0, \quad\left|\lambda s^{-\alpha}\right|<1 .
$$

An effective way to compute $e_{\alpha, \beta}(t ; \lambda)$ is by approximating the inversion Laplace transform formula as follows:

$$
e_{\alpha, \beta}(t ; \lambda)=\frac{1}{2 \pi i} \int_{\mathcal{C}} e^{s t} \mathcal{E}_{\alpha, \beta}(s ; \lambda) \mathrm{d} s,
$$

where contour $\mathcal{C}$ is a suitable deformation of the Bromwich line. Based on this approach, the corresponding MATLAB code is made freely available (cf. [33]).

The following results on the integration of $e_{\alpha, \beta}$ function will be used later (cf. [2]).

Lemma 2.1. Suppose that $t \geq 0, \Re(\alpha)>0$, and $\beta$ $>0$, and let $r \in \mathbb{R}$ be such that $r>-1$. Then:

$$
\int_{0}^{t} e_{\alpha, \beta}(t-s ; \lambda) s^{r} \mathrm{~d} s=\Gamma(r+1) e_{\alpha, \beta+r+1}(t ; \lambda) .
$$

Lemma 2.2. Suppose that $a<b \leq t, \Re(\alpha)>0$, and $\beta>0$. Then:

$$
\int_{a}^{b} e_{\alpha, \beta}(t-s ; \lambda) \mathrm{d} s=e_{\alpha, \beta+1}(t-a ; \lambda)-e_{\alpha, \beta+1}(t-b ; \lambda),
$$

and:

$$
\begin{aligned}
& \int_{a}^{b} e_{\alpha, \beta}(t-s ; \lambda)(s-a) \mathrm{d} s=e_{\alpha, \beta+2}(t-a ; \lambda) \\
& \quad-(b-a) e_{\alpha, \beta+1}(t-b ; \lambda)-e_{\alpha, \beta+2}(t-b ; \lambda) .
\end{aligned}
$$

This section ends with a brief discussion of linear FDEs. Let us consider the following initial value problem, on $0<\alpha<1$, containing a linear FDEs with constant coefficients:

$$
D_{*}^{\alpha} u(t)=\lambda u(t)+g(t), \quad u(0)=u_{0},
$$

where $u(t):[0, T] \rightarrow \mathbb{R}$ and source term $g(t)$ is assumed to be sufficiently smooth. Using the Laplace transform (cf. [2, (2.253)]), the desired solution to Problem (5) can be expressed as follows:

$$
u(t)=e_{\alpha, 1}(t ; \lambda) u_{0}+\int_{0}^{t} e_{\alpha, \alpha}(t-s ; \lambda) g(s) \mathrm{d} s .
$$

\subsection{Use of matrix functions}

Let $A$ be a real or complex square matrix of order $m$. From elementary linear algebra, there exists nonsingular matrix $Z$, such that:

$$
Z^{-1} A Z=\operatorname{diag}\left(J_{1}, J_{2}, \cdots, J_{p}\right),
$$

where $J_{k}$ is called a Jordan block of size $m_{k}$ with eigenvalue $\lambda_{k}$ of algebraic multiplicity $m_{k}$ and $m_{1}+$ $m_{2}+\cdots+m_{p}=m$. Suppose that function $f$ is defined on the spectrum of $A$, then:

$$
f(A)=Z \operatorname{diag}\left(f\left(J_{1}\right), f\left(J_{2}\right), \cdots, f\left(J_{p}\right)\right) Z^{-1},
$$

where $f\left(J_{k}\right)$ is the upper triangular Toeplitz matrix defined as follows: Writing $J_{k}=\lambda_{k} I+N_{k}$ where $N_{k}$ 
is its strictly upper bi-diagonal part by the obtained Taylor series expansion:

$f\left(J_{k}\right)=f\left(\lambda_{k}\right) I+f^{\prime}\left(\lambda_{k}\right) N_{k}+\cdots+\frac{f^{\left(m_{k}-1\right)}\left(\lambda_{k}\right)}{\left(m_{k}-1\right) !} N_{k}^{m_{k}-1}$,

since all powers of nilpotent matrix $N_{k}$ from the $m_{k}$ th onwards are zero (cf. [14]).

In the case of diagonalizable matrices, the problem becomes very simple and Jordan form (6) reduces to an eigenvalue decomposition:

$$
A=X \Lambda X^{-1},
$$

where $\Lambda=\operatorname{diag}\left(\lambda_{1}, \lambda_{2}, \cdots, \lambda_{m}\right)$ and the columns of matrix $X \in \mathbb{C}^{m \times m}$ contain linearly independent eigenvectors of $A$. Then:

$$
f(A)=X \operatorname{diag}\left(f\left(\lambda_{1}\right), f\left(\lambda_{2}\right), \cdots, f\left(\lambda_{m}\right)\right) X^{-1},
$$

and hence, just the values of function $f$ on scalar arguments $\lambda$ have to be computed.

\section{Problem statement}

The structural equations of motion differ from the classical formulations as fractional order derivatives are used to model the viscoelastic-damping phenomenon (cf. [34]). Bagley commonly attributes the beginning of the modern uses of fractional calculus in linear viscoelasticity to the $1979 \mathrm{PhD}$ thesis under supervision of Professor Torvik (cf. $[5,35]$ ). To construct the desired model, the main ideas developed in $[2,15]$ will be followed.

A Newtonian fluid with density $\rho$ and viscosity $\mu$, initially at rest, is considered, and it permits the plate at the boundary to commence a general transverse motion. Let $\sigma(t, z)$ and $v(t, z)$ be the stress and transverse fluid velocity fields, respectively, which are functions of time $t$ and distance $z$ from the fluid-plate contact boundary. It is known that (cf. $[15,(13)]$ ) the unusual relationship between $\sigma(t, z)$ and $v(t, z)$ is as follows:

$$
\sigma(t, z)=\sqrt{\mu \rho} D_{*}^{1 / 2} v(t, z) .
$$

The physical interpretation of Relationship (8) is that stress at a given point at any time is dependent on the time history of velocity profile at that point.

Let us now consider a rigid plate of mass $M$ and area $S$ immersed in a Newtonian fluid of infinite extent and connected by a massless spring of stiffness $K$ to a fixed point. The system is depicted in Figure 1. Force $f(t)$ is applied to the plate, and we find the differential equation describing displacement $y(t)$ of the plate to be:

$$
M y^{\prime \prime}(t)=f(t)-K y(t)-2 S \sigma(t, 0) .
$$

Substituting the stress from Eq. (8) and using $v(t, 0)=$

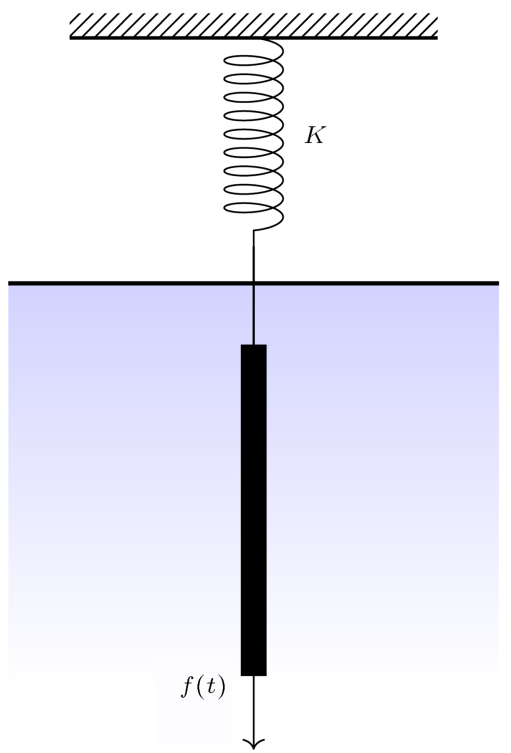

Figure 1. An immersed plate in a Newtonian fluid [2].

$y^{\prime}(t)$, the following FDE is obtained:

$$
M y^{\prime \prime}(t)+2 S \sqrt{\mu \rho} D_{*}^{3 / 2} y(t)+K y(t)=f(t), \quad t>0 .
$$

A similar equation according to the Riemann-Liouville approach can be obtained. In this case, displacements $y(t)$ and velocities $v(t, 0)$ of the plate fluid system must be initially zero (cf. $[2,(8.19)])$.

As usual, equations of this type are called BagleyTorvik equation $[2,17]$. This paper deals with the numerical solution to the following Bagley-Torvik equation:

$$
y^{\prime \prime}(t)+b D_{*}^{3 / 2} y(t)+c y(t)=f(t), \quad 0 \leq t \leq T,
$$

with the initial conditions:

$$
y(0)=Y_{0}, \quad y^{\prime}(0)=Y_{0}^{\prime},
$$

where $Y_{0}$ and $Y_{0}^{\prime}$ are arbitrary real numbers. An analytical solution for homogeneous initial conditions $\left(Y_{0}=Y_{0}^{\prime}=0\right)$ can be given in the form of:

$$
y(t)=\int_{0}^{t} G(t-s) f(s) \mathrm{d} s,
$$

with Green function:

$$
G(t)=\sum_{k=0}^{\infty} \frac{(-1)^{k}}{k !} c^{k} t^{2 k+1} E_{1 / 2,2+3 k / 2}^{(k)}(-b \sqrt{t})
$$

where $E_{\alpha, \beta}^{(k)}$ is the $k$ th derivative of the ML function (cf. $[2,(8.26)])$ given by:

$$
E_{\alpha, \beta}^{(k)}(z)=\sum_{j=0}^{\infty} \frac{(j+k) ! z^{j}}{j ! \Gamma(\alpha j+\alpha k+\beta)} .
$$

This analytical solution includes the evaluation of a convolution integral, which is comprised of a Green's 
function stated as an infinite sum of derivatives of ML functions, and this cannot be evaluated easily for general functions $f$. For inhomogeneous initial conditions, expressions that are even more sophisticated arise (cf. [17]). The difficulty of obtaining an analytical solution was a motivation to investigate numerical schemes for the solution of Eq. (9) with initial conditions Eqs. (10) under which it can be performed more efficiently.

Before coming to the description of our numerical scheme, it would be convenient to rewrite the original Bagley-Torvik equation (Eq. (9)) in the form of a system of FDEs of order $1 / 2$ that will later be solved numerically. In particular, the system to be considered is of the following form:

$$
\begin{aligned}
& D_{*}^{1 / 2} y_{0}(t)=y_{1}(t), \\
& D_{*}^{1 / 2} y_{1}(t)=y_{2}(t), \\
& D_{*}^{1 / 2} y_{2}(t)=y_{3}(t), \\
& D_{*}^{1 / 2} y_{3}(t)=-c y_{0}(t)-b y_{3}(t)+f(t),
\end{aligned}
$$

with the initial conditions:

$$
\begin{array}{ll}
y_{0}(0)=Y_{0}, & y_{1}(0)=0, \\
y_{2}(0)=Y_{0}^{\prime}, & y_{3}(0)=0 .
\end{array}
$$

In this context, a useful result, which can finally help us implement EIs, is presented in the following Theorem (cf. [17]).

Theorem 3.1. The Bagley-Torvik (Eq. (9)) with initial conditions (Eqs. (10)) is equivalent to the system of Eqs. (12) together with the initial conditions (Eqs. (13)) in the following sense:

1. Whenever $\left[y_{0}, y_{1}, y_{2}, y_{3}\right]^{T}$ with $y_{0} \in \mathcal{C}^{2}[0, T]$ for some $T>0$ is the solution to the initial value problems (Eqs. (12) and (13)), function $y:=y_{0}$ solves the initial value problem (9)-(10);

2. Whenever $y \in \mathcal{C}^{2}[0, T]$ is a solution to the initial value problem (9)-(10), vector-valued function $\mathbf{Y}:=$ $\left[y_{0}, y_{1}, y_{2}, y_{3}\right]^{T}:=\left[y, D_{*}^{1 / 2} y, y^{\prime}, D_{*}^{3 / 2} y\right]^{T}$ satisfies the initial value problem (12)-(13).

Thus, an equation of the following type can be obtained:

$$
D_{*}^{1 / 2} \mathbf{Y}(t)=A \mathbf{Y}(t)+\mathbf{F}(t), \quad \mathbf{Y}(0)=\mathbf{Y}_{0}
$$

with a given vector-valued function $\mathbf{F}(t)=[0,0,0$, $f(t)]^{T}$, unknown solution $\mathbf{Y}$, initial condition vector
$\mathbf{Y}_{0}=\left[Y_{0}, 0, Y_{0}^{\prime}, 0\right]^{T}$, and $4 \times 4$ matrix $A$ of constants are as follows:

$$
A=\left[\begin{array}{cccc}
0 & 1 & 0 & 0 \\
0 & 0 & 1 & 0 \\
0 & 0 & 0 & 1 \\
-c & 0 & 0 & -b
\end{array}\right]
$$

It would be interesting to justify the reason why $y_{1}(0)$ and $y_{3}(0)$ can be assumed equal to 0 . Indeed, by means of Eqs. (2) and (12), the following results will be obtained:

$$
\begin{aligned}
& y_{1}(t)=D_{*}^{1 / 2} y_{0}(t)=J_{0}^{1 / 2} y^{\prime}(t), \\
& y_{3}(t)=D_{*}^{3 / 2} y_{0}(t)=J_{0}^{1 / 2} y^{\prime \prime}(t) .
\end{aligned}
$$

Since $y^{\prime}$ and $y^{\prime \prime}$ are continuous functions, integrals $J_{0}^{1 / 2} y^{\prime}(t)$ and $J_{0}^{1 / 2} y^{\prime \prime}(t)$ vanish for $t \rightarrow 0(\mathrm{cf}$. [17, Lemma 2.2]).

Hence, by an argument similar to that used for scalar case (Eq. (5)), the exact solution of Eq. (14) at time $t$ is provided by the familiar variation-of-constant formula:

$$
\mathbf{Y}(t)=e_{1 / 2,1}(t ; A) \mathbf{Y}_{0}+\int_{0}^{t} e_{1 / 2,1 / 2}(t-s ; A) \mathbf{F}(s) \mathrm{d} s .
$$

Formula (15) of the exact solution of Eq. (14) is usually considered just as a theoretical tool and is disregarded for practical computation due to the difficulty of integration and of evaluating the ML function with matrix arguments. Therefore, numerical solution is the best approach to overcoming these difficulties.

\section{Exponential integrators for fractional problem}

Constructing a class of fractional EIs for Eq. (14) in the spirit of $[10,11,12]$ can be the starting point. The general idea can be as follows. For simplicity, uniform grid points $t_{n}=n h, n=0,1, \cdots$, where $h>0$ is the step size is assumed. Eq. (15) is written in the piecewise manner as follows:

$$
\begin{aligned}
\mathbf{Y}\left(t_{n}\right)= & e_{1 / 2,1}\left(t_{n} ; A\right) \mathbf{Y}_{0} \\
& +\sum_{j=0}^{n-1} \int_{t_{j}}^{t_{j+1}} e_{1 / 2,1 / 2}\left(t_{n}-s ; A\right) \mathbf{F}(s) \mathrm{d} s .
\end{aligned}
$$

An EI results from Eq. (16) if $\mathbf{F}(s)$ is expressed approximately by a linear combination of values at some grid points selected from interval $\left[t_{0}, t_{j+1}\right]$. A simple way to do this is to approximate $\mathbf{F}(s)$ by the unique polynomial interpolating $\mathbf{F}(s)$ at such points. This will be implemented in two different ways, which 
do not suffer from restrictions on the step-size due to stability requirements. It has been proved that the stability of this approach is unconditionally guaranteed for methods based on polynomials of degree not greater than 2 (cf. [36]). The resulting discretization scheme involves the evaluation of ML functions on matrix arguments and is performed after diagonalizing the matrix of the system. Due to the small dimension of the systems, the latter task does not appear to be illconditioned.

\subsection{Fractional exponential Euler scheme}

In this section, a fairly simple algorithm for numerical computation of Eq. (16) is described. In each subinterval $\left[t_{j}, t_{j+1}\right]$, vector field $\mathbf{F}(s)$ is approximated by constant value $\mathbf{F}_{j}:=\mathbf{F}\left(t_{j}\right)$. The application of Lemma 2.2 into Eq. (16) leads to:

$$
\mathbf{Y}_{n}=e_{1 / 2,1}\left(t_{n} ; A\right) \mathbf{Y}_{0}+\sum_{j=0}^{n-1} W_{n, j} \mathbf{F}_{j}
$$

where $\mathbf{Y}_{n}$ stands for the approximation of $\mathbf{Y}\left(t_{n}\right)$ and weights $W_{n, j}$ are matrix functions defined as follows:

$$
W_{n, j}=e_{1 / 2,3 / 2}\left(t_{n}-t_{j} ; A\right)-e_{1 / 2,3 / 2}\left(t_{n}-t_{j+1} ; A\right) .
$$

The evaluation of weights $W_{n, j}$ in EI (Eq. (17)) involves the computation of ML functions with matrix arguments. It is well known that this is not a trivial task.

A simple implementation of Eq. (17) is proposed. Given $A$ in its eigenvalue decomposition (7), after putting $\mathbf{Z}_{j}=X^{-1} \mathbf{Y}_{j}$ and $\mathbf{G}_{j}=X^{-1} \mathbf{F}_{j}$, Eq. (17) can be equivalently written as $\mathbf{Y}_{n}=X \mathbf{Z}_{n}$ with:

$$
\begin{gathered}
\mathbf{Z}_{n}=e_{1 / 2,1}\left(t_{n} ; \Lambda\right) \mathbf{Z}_{0}+W_{n, 0} \mathbf{G}_{0}+\sum_{j=1}^{n-1} W_{n, j} \mathbf{G}_{j} \\
=e_{1 / 2,1}(n h ; \Lambda) \mathbf{Z}_{0}+e_{1 / 2,3 / 2}(n h ; \Lambda) \mathbf{G}_{0} \\
\quad+\sum_{j=1}^{n-1} e_{1 / 2,3 / 2}((n-j) h ; \Lambda) \nabla \mathbf{G}_{j}
\end{gathered}
$$

where $\nabla \mathbf{G}_{j}=\mathbf{G}_{j}-\mathbf{G}_{j-1}$ are the classical backward differences. Then, Eq. (4) yields:

$$
\mathbf{Z}_{n}=e_{1 / 2,1}(n ; \mathrm{D}) \mathbf{Z}_{0}+h^{1 / 2} \sum_{j=0}^{n-1} W_{n-j}^{(1)} \nabla \mathbf{G}_{j},
$$

with $\nabla \mathbf{G}_{0}=\mathbf{G}_{0}, \mathrm{D}=h^{1 / 2} \Lambda$, and:

$$
W_{n}^{(1)}=e_{1 / 2,3 / 2}(n ; \mathrm{D}) \text {. }
$$

4.2. Fractional exponential trapezoidal scheme Given Eq. (16), the scheme can be obtained by employing the piecewise first-order interpolating polynomials:

$$
\mathbf{P}_{1}(s):=\mathbf{F}_{j}+\frac{1}{h}\left(s-t_{j}\right) \nabla \mathbf{F}_{j+1},
$$

and replacing $\mathbf{F}(s)$ by $\mathbf{P}_{1}(s)$ when $s \in\left[t_{j}, t_{j+1}\right]$. Then:

$$
\begin{aligned}
\mathbf{Y}_{n}= & e_{1 / 2,1}\left(t_{n} ; A\right) \mathbf{Y}_{0} \\
& +\sum_{j=0}^{n-1} \int_{t_{j}}^{t_{j+1}} e_{1 / 2,1 / 2}\left(t_{n}-s ; A\right) \mathbf{F}_{j} \mathrm{~d} s \\
& +\frac{1}{h} \sum_{j=0}^{n-1} \int_{t_{j}}^{t_{j+1}} e_{1 / 2,1 / 2}\left(t_{n}-s ; A\right)\left(s-t_{j}\right) \nabla \mathbf{F}_{j+1} \mathrm{~d} s .
\end{aligned}
$$

As before, by using Lemma 2.2, the exponential trapezoidal scheme can be obtained as follows:

$$
\begin{aligned}
\mathbf{Y}_{n}= & e_{1 / 2,1}\left(t_{n} ; \Lambda\right) \mathbf{Y}_{0} \\
& +\frac{1}{h}\left(\widetilde{W}_{0} \mathbf{F}_{0}+\sum_{j=1}^{n-1} \widetilde{W}_{n, j} \mathbf{F}_{j}+\widetilde{W}_{n} \mathbf{F}_{n}\right),
\end{aligned}
$$

with weights:

$$
\begin{aligned}
\widetilde{W}_{0}= & e_{1 / 2,5 / 2}\left(t_{n-1} ; A\right)+h e_{1 / 2,3 / 2}\left(t_{n} ; A\right) \\
& -e_{1 / 2,5 / 2}\left(t_{n} ; A\right), \\
\widetilde{W}_{n, j}= & e_{1 / 2,5 / 2}\left(t_{n}-t_{j+1} ; A\right)-2 e_{1 / 2,5 / 2}\left(t_{n}-t_{j} ; A\right) \\
& +e_{1 / 2,5 / 2}\left(t_{n}-t_{j-1} ; A\right), \\
\widetilde{W}_{n}= & e_{1 / 2,5 / 2}\left(t_{1} ; A\right) .
\end{aligned}
$$

The resulting explicit scheme is given for $n \geq 2$ by:

$$
\begin{aligned}
\mathbf{Z}_{n}= & e_{1 / 2,1}(n ; \mathrm{D}) \mathbf{Z}_{0} \\
& +h^{1 / 2}\left(W^{(2)} \mathbf{G}_{0}+\sum_{j=1}^{n-1} W_{n-j}^{(2)} \mathbf{G}_{j}+W_{0}^{(2)} \mathbf{G}_{n}\right)
\end{aligned}
$$

where, as before, $\mathrm{D}=h^{1 / 2} \Lambda$ and:

$$
W_{n}^{(2)}= \begin{cases}e_{1 / 2,5 / 2}(1 ; \mathrm{D}) & n=0, \\ e_{1 / 2,5 / 2} & (n-1 ; \mathrm{D}) \\ -2 e_{1 / 2,5 / 2}(n ; \mathrm{D}) & n \geq 1 . \\ +e_{1 / 2,5 / 2}(n+1 ; \mathrm{D}) & \end{cases}
$$

and:

$$
\begin{aligned}
W^{(2)}= & e_{1 / 2,5 / 2}(n-1 ; \mathrm{D})+e_{1 / 2,3 / 2}(n ; \mathrm{D}) \\
& -e_{1 / 2,5 / 2}(n ; \mathrm{D}) .
\end{aligned}
$$




\subsection{A particular case}

Here, a particular case of right-hand side function, $f(t)$, in Eq. (9) can be considered, where $f(t)$ can be expressed in terms of powers of $t$. Indeed, given that:

$$
f(t)=\sum_{\nu \in \mathcal{J}} c_{\nu} t^{\nu}
$$

with $\mathcal{J} \subset\{\nu \in \mathbb{R}, \nu>-1\}$ an index set and $c_{\nu}$ some real coefficients the exact solution of System (14) can be expressed by means of Lemma 2.1 as follows:

$$
\begin{aligned}
\mathbf{Y}(t)= & e_{1 / 2,1}(t ; A) \mathbf{Y}_{0} \\
& +\sum_{\nu \in \mathcal{J}} c_{\nu} \Gamma(\nu+1) e_{1 / 2,3 / 2+\nu}(t ; A) \mathbf{e}_{4} .
\end{aligned}
$$

Here, $\mathbf{e}_{4}$ is the fourth vector of the canonical basis of $\mathbb{R}^{4}$. One of the most obvious advantages of exploiting Eq. (21) for practical computation is overcoming stability issues, and the problem is indeed solved in an almost exact way with providing satisfactory results in the presence of stiffness as well. Another important success related to the proposed approach is that the numerical solution can be directly evaluated at any given time, $t$, without the need for approximating the corresponding values at previous time points.

\section{Error analysis}

In this section, the convergence of the fractional Exponential Euler Scheme (EES) with the fractional Exponential Trapezoidal Scheme (ETS) is proved. In addition, the attainable order of convergence is studied. To express this theme, first, the following auxiliary lemma is presented (cf. [37]).

Lemma 5.1. $\operatorname{Let} g(x)=(1-x)^{\beta} k(x)$, where $\beta>-1$ and $k \in \mathcal{C}([0,1])$. The general trapezoidal quadrature rule:

$$
Q_{n}^{\nu}[g]=\frac{1}{n} \sum_{j=1}^{n} g\left(\frac{2 j-1+\nu}{2 n}\right), \quad|\nu|<1,
$$

approximates integral $I[g]=\int_{0}^{1} g(t) d t$ with an error:

$$
Q_{n}^{\nu}[g]-I[g]=-\frac{\nu}{2} k(0) n^{-1}+\mathcal{O}\left(n^{-\beta-1}\right) .
$$

The convergence of fractional EES can be proved by the same kind of ideas developed in $[10,11]$.

Theorem 5.2. Let $f \in \mathcal{C}^{1}([0, T])$. Then, the numerical solution provided by the fractional EES converges with order $p=1$. More precisely, error
$\mathbf{E}_{n}:=\mathbf{Y}_{n}-\mathbf{Y}\left(t_{n}\right)$ of fractional EES (Eq. (17)) is expressed as:

$$
\begin{aligned}
\mathbf{E}_{n} & =\frac{h}{2} e_{1 / 2,3 / 2}\left(t_{n}, A\right) \mathbf{F}^{\prime}\left(\eta t_{n}\right)+\mathcal{O}\left(h^{3 / 2}\right), \\
\eta & \in(0,1) .
\end{aligned}
$$

Proof. Subtract the true solution in Eq. (16) from Eq. (17), so the error can be written as follows:

$$
\mathbf{E}_{n}=\sum_{j=0}^{n-1} \int_{t_{j}}^{t_{j+1}} e_{1 / 2,1 / 2}\left(t_{n}-s ; A\right)\left(\mathbf{F}_{j}-\mathbf{F}(s)\right) \mathrm{d} s .
$$

Applying the change of variable $s=t_{j}+\theta h$, scaling Eq. (4) and the Taylor's theorem, the error is obtained as follows:

$$
\begin{aligned}
\mathbf{E}_{n}= & h^{3 / 2} \sum_{j=0}^{n-1} \int_{0}^{1} e_{1 / 2,1 / 2}(n-j-\theta ; A)(1-\theta) \\
& \mathbf{F}^{\prime}\left(t_{j}+\theta h\right) \mathrm{d} \theta+\mathcal{O}\left(h^{5 / 2}\right) .
\end{aligned}
$$

Using the series expansion defining the ML function:

$$
\begin{aligned}
\mathbf{E}_{n}= & h^{3 / 2} \sum_{k=0}^{\infty} \frac{h^{k / 2} A^{k}}{\Gamma(\beta+1)} \int_{0}^{1}(1-\theta) \sum_{j=0}^{n-1}(n-j-\theta)^{\beta} \\
& \mathbf{F}^{\prime}\left(t_{j}+\theta h\right) \mathrm{d} \theta+\mathcal{O}\left(h^{5 / 2}\right),
\end{aligned}
$$

where $\beta=(k-1) / 2$. Using Lemma 5.1 the following result is obtained:

$$
\begin{aligned}
& \sum_{j=0}^{n-1}(n-j-\theta)^{\beta} \mathbf{F}^{\prime}\left(t_{j}+\theta h\right) \\
& =n^{\beta} \sum_{j=1}^{n}\left(1-\frac{j-1+\theta}{n}\right)^{\beta} \mathbf{F}^{\prime}((j-1+\theta) h) \\
& =n^{\beta+1} \int_{0}^{1}(1-\tau)^{\beta} \mathbf{F}^{\prime}\left(t_{n} \tau\right) \mathrm{d} \tau \\
& \quad+n^{\beta}\left(\frac{1}{2}-\theta\right) \mathbf{F}^{\prime}(0)+\mathcal{O}(1)
\end{aligned}
$$

Applying the integral term mean value theorem to the integral term, the following result is obtained:

$$
\begin{aligned}
& \sum_{j=0}^{n-1}(n-j-\theta)^{\beta} \mathbf{F}^{\prime}\left(t_{j}+\theta h\right)=\frac{n^{\beta+1}}{\beta+1} \mathbf{F}^{\prime}\left(\eta t_{n}\right) \\
& +n^{\beta}\left(\frac{1}{2}-\theta\right) \mathbf{F}^{\prime}(0)+\mathcal{O}(1),
\end{aligned}
$$

with $\eta \in(0,1)$. Thus, the expansion of error $\mathbf{E}_{n}$ can be written as follows: 


$$
\begin{aligned}
\mathbf{E}_{n} & =\frac{h}{2} \sum_{k=0}^{\infty} \frac{t_{n}^{(k+1) / 2} A^{k}}{\Gamma((k+3) / 2)} \mathbf{F}^{\prime}\left(\eta t_{n}\right) \\
& +\frac{h^{2}}{12} \sum_{k=0}^{\infty} \frac{t_{n}^{(k-1) / 2} A^{k}}{\Gamma((k+1) / 2)} \mathbf{F}^{\prime}(0)+\mathcal{O}\left(h^{3 / 2}\right) \\
& =\frac{h}{2} e_{1 / 2,3 / 2}\left(t_{n} ; A\right) \mathbf{F}^{\prime}\left(\eta t_{n}\right) \\
& +\frac{h^{2}}{12} e_{1 / 2,1 / 2}\left(t_{n} ; A\right) \mathbf{F}^{\prime}(0)+\mathcal{O}\left(h^{3 / 2}\right),
\end{aligned}
$$

and the proof is complete.

Corollary 5.3. Suppose that $f \in \mathcal{C}^{1}([0, T])$ and $y_{n}$, $n=1, \cdots, N$, as the numerical solution provided by fractional EES. Then:

$$
\left|y\left(t_{n}\right)-y_{n}\right| \leq C h, \quad n=1, \cdots, N,
$$

with $N=\lfloor T / h\rfloor$ and $C$ as a suitable constant which does not depend on $h$ and $n$.

A similar error estimation procedure for the solution of fractional ETS can be currently proposed.

Theorem 5.4. Let $f \in \mathcal{C}^{2}([0, T])$. Then, the numerical solution provided by the fractional ETS converges with order $p=2$. More precisely, error $\mathbf{E}_{n}:=\mathbf{Y}_{n}-\mathbf{Y}\left(t_{n}\right)$ of fractional ETS (Eq. (19)) is expressed as follows:

$$
\begin{aligned}
\mathbf{E}_{n} & =\frac{h^{2}}{12} e_{1 / 2,3 / 2}\left(t_{n}, A\right) \mathbf{F}^{\prime \prime}\left(\eta t_{n}\right)+\mathcal{O}\left(h^{5 / 2}\right), \\
\eta & \in(0,1) .
\end{aligned}
$$

It should be noted that the proof of Theorem 5.2 can be adopted without difficulty to deal with Theorem 5.4.

Corollary 5.5. Suppose that $f \in \mathcal{C}^{2}([0, T])$ and $y_{n}$, $n=1, \cdots, N$, as the numerical solution provided by fractional ETS. Then:

$$
\left|y\left(t_{n}\right)-y_{n}\right| \leq C h^{2}, \quad n=1, \cdots, N,
$$

with $N=\lfloor T / h\rfloor$ and $C$ as a suitable constant which does not depend on $h$ and $n$.

This section ends with a brief discussion of the less regular case in which the source term is $f \equiv$ $f(y(t))$. The resulting system is a semi-linear problem as follows:

$$
D_{*}^{\alpha} \mathbf{Y}(t)=A \mathbf{Y}(t)+\mathbf{F}(\mathbf{Y}(t)), \quad \mathbf{Y}(0)=\mathbf{Y}_{0}
$$

According to the results in [10], by applying fractional ETS, one should obtain an order of convergence proportional to $h^{3 / 2}$ and not to $h^{2}$ as stated in the case $f \equiv f(t)$ in Theorem 5.4. Indeed, a drop in the order of convergence is expected, since $y(t)$ and $f(y(t))$ are usually singular at the origin. Thus, the main advantage of the use of EIs for FDEs is indeed not just confined to stability, but is also related to the improvement of the order of convergence when linear systems are solved.

\section{Results and discussion}

In this section, some numerical studies are presented to illustrate and test the behavior of the approach described in Section 4. All the algorithms are implemented in Matlab and the evaluation of scalar ML function on the spectrum of coefficient matrix $A$ is performed by the Matlab code $\mathrm{ml}$ from [33]. Since we were originally interested in the solution of the given scalar the initial value problem (9)-(10), there is a need to look at the first component of solution vector $\mathbf{Y}$.

Example 1. As a first example, we choose $f(t)$ so that the exact solution to the initial value problems (9)(10) is $y(t)=t^{\gamma}$. It can be checked that the corresponding forcing term is as follows:

$$
f(t)=\gamma(\gamma-1) t^{\gamma-2}+\frac{b \Gamma(\gamma+1)}{\Gamma(\gamma-1 / 2)} t^{\gamma-3 / 2}+c t^{\gamma} .
$$

The numerical solution is evaluated at $t=1$ according to the fractional EES and the fractional ETS by a sequence of decreasing step size $h$. Errors $e(h)$, with respect to the exact solution, together with an experimental order of convergence, denoted with EOC and obtained as $\log _{2}(e(h) / e(h / 2))$, are reported in Table 1. The close agreement of the empirical values of EOC with the theoretically predicted values of 1 and 2 can be seen, respectively. In addition, the absolute errors are compared with the Podlubny's Matrix Approach (PMA) in [23].

Example 2. The initial value problem (9)-(10) with $b=\beta \sqrt{\pi}$ are considered, in which $\beta$ is a parameter, $c=1, Y_{0}=1, Y_{0}^{\prime}=0$, and $f(t) \equiv 0$. This mathematical model is developed for a micro-electromechanical system instrument, designed primarily to measure the viscosity of fluids encountered during oil well exploration. As mentioned in [38], the solution reduces to $\cos t$, as $\beta \rightarrow 0$, as illustrated. Since $\mathbf{F} \equiv \mathbf{0}$, then $\mathbf{Y}(t)=e_{1 / 2,1}(t ; A) \mathbf{Y}_{0}$, by means of Eq. (15). The numerical solutions for various parameter $\beta$ and $T=30$ are plotted in Figure 2 and are reported in Table 2.

Example 3. The most popular initial value problem (9)-(10) can be assumed with $Y_{0}=Y_{0}^{\prime}=0$ and:

$$
f(t)= \begin{cases}8 & 0 \leq t \leq 1 \\ 0 & t>1\end{cases}
$$


Table 1. The resulting values of errors, EOC, and PMA at $t=1$ for $b=c=1$ (Example 1).

\begin{tabular}{cccccccc}
\hline \multirow{2}{*}{$\boldsymbol{h}$} & \multicolumn{3}{c}{$\boldsymbol{\gamma}=\mathbf{3}$} & & \multicolumn{3}{c}{$\boldsymbol{\gamma}=\mathbf{4}$} \\
\cline { 2 - 3 } & Error & EOC & PMA [23] & & Error & EOC & PMA [23] \\
\hline $1 / 8$ & $1.76(-01)$ & & $2.77(-01)$ & & $1.57(-02)$ & & $4.76(-01)$ \\
$1 / 16$ & $9.09(-02)$ & 0.956 & $1.50(-01)$ & & $3.91(-03)$ & 2.011 & $2.31(-01)$ \\
$1 / 32$ & $4.62(-02)$ & 0.978 & $7.76(-02)$ & & $9.77(-04)$ & 2.003 & $1.13(-01)$ \\
$1 / 64$ & $2.33(-02)$ & 0.989 & $3.94(-02)$ & & $2.44(-04)$ & 2.000 & $5.58(-02)$ \\
$1 / 128$ & $1.17(-02)$ & 0.994 & $1.98(-02)$ & & $6.10(-05)$ & 2.000 & $2.77(-02)$ \\
$1 / 256$ & $5.85(-03)$ & 0.997 & $9.96(-03)$ & & $1.53(-05)$ & 2.000 & $1.38(-02)$ \\
$1 / 512$ & $2.93(-03)$ & 0.999 & $4.99(-03)$ & & $3.81(-06)$ & 2.000 & $6.90(-03)$ \\
\hline
\end{tabular}

Table 2. The resulting values of EI with $\beta=0.20$ in some values of $t$ (Example 2).

\begin{tabular}{ccccccccc}
\hline $\boldsymbol{t}$ & $\mathbf{1}$ & $\mathbf{2}$ & $\mathbf{5}$ & $\mathbf{1 0}$ & $\mathbf{1 5}$ & $\mathbf{2 0}$ & $\mathbf{2 5}$ & $\mathbf{3 0}$ \\
\hline$y(t)$ & 0.6188 & -0.1398 & -0.2436 & -0.3114 & 0.2086 & 0.0330 & -0.0936 & 0.0229 \\
\hline
\end{tabular}

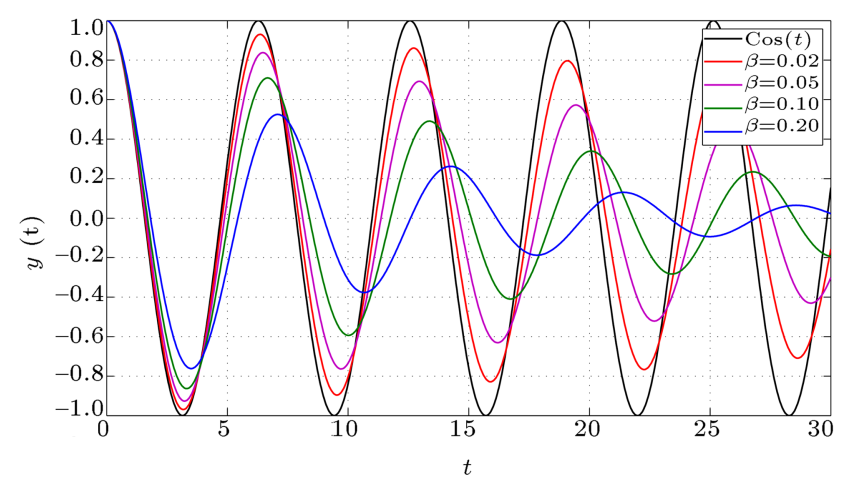

Figure 2. Fractional Bagley-Torvik equation (Example 2).

Since $\mathbf{Y}_{0} \equiv \mathbf{0}$ by means of Eq. (15) and Lemma 2.2, $\mathbf{Y}(t)=8 B(t) \mathbf{e}_{4}$, where:

$$
B(t)= \begin{cases}e_{1 / 2,3 / 2}(t ; A) & 0 \leq t \leq 1, \\ e_{1 / 2,3 / 2}(t ; A)-e_{1 / 2,3 / 2}(t-1 ; A) & t>1 .\end{cases}
$$

Thanks to the work of Črmák and Kisela in [16], the asymptotic behavior of the exact solution is $y(t)=$ $\mathcal{O}\left(t^{-1 / 2}\right)$ as $t \rightarrow \infty$. In Figure 3 , the first component of the solution vector $\mathbf{Y}(t)$ for $b=c=0.5, b=c=1$, and $T=30$ are illustrated. The resulting values of the present method and some numerical methods for $t=1,2, \cdots, 10$ are also reported in Table 3 . It can be seen that the presented method provides closer results to the exact solution than others.

Example 4. The last test is initial value problems (Eqs. (9) and (10)) with $b=2, c=1, Y_{0}=0, Y_{0}^{\prime}=$ 1 , and $f(t)=\sin t$. Unfortunately, an exact solution to these problems is not available. Thus, a reference solution, the numerical approximation given by PMA in [23] with step sizes $h=0.01$, is used. The numerical

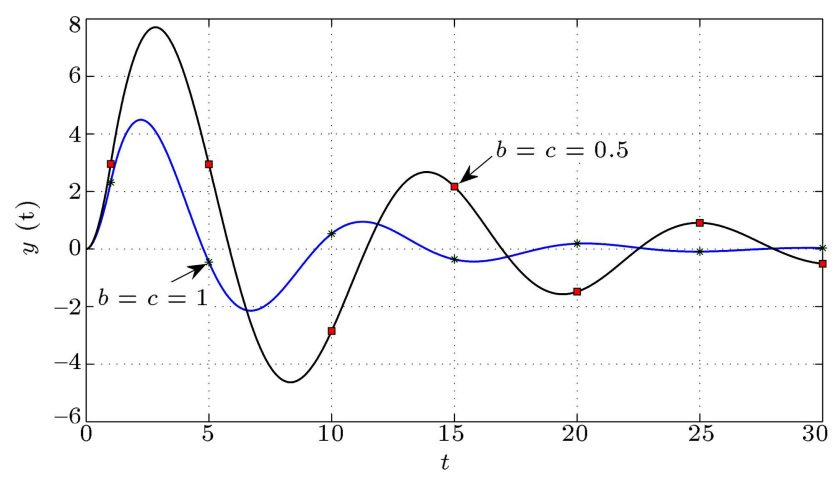

Figure 3. Fractional Bagley-Torvik equation: solution by EI (Example 3.)

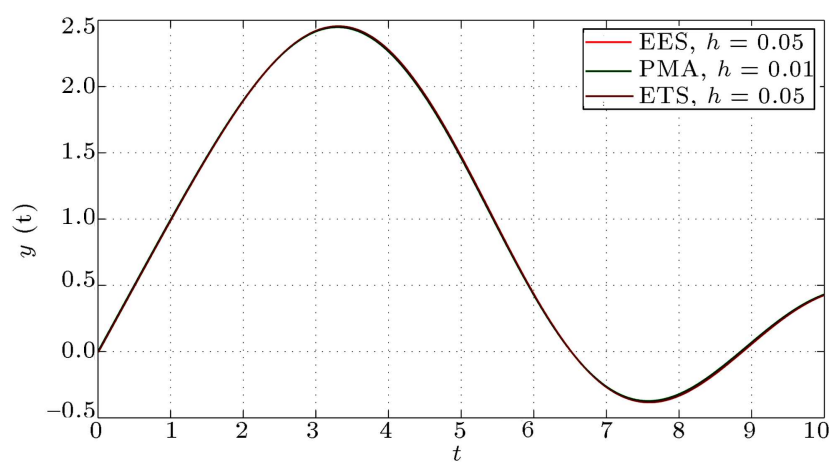

Figure 4. Numerical solution by EIs and PMA (Example 4).

results of the new method proposed in this paper and PMA with $T=10$ are shown in Figure 4 and in Table 4 . These results indicate that the approximate solutions of the present method are in agreement with those of the literature reviews.

\section{Conclusions}

This paper deals with a numerical method to solve the Bagley-Torvik equation using the EIs method. In 
Table 3. The resulting values of EI and available methods with $b=c=0.5$ (Example 3).

\begin{tabular}{cccccc}
\hline $\boldsymbol{t}$ & EI & HWM [20] & HFA [18] & PMA [23] & Exact (11) \\
\hline 1 & 2.9526 & 3.5386 & 2.9526 & 2.9182 & 2.952583 \\
2 & 6.7601 & 7.5372 & 6.7602 & 6.6732 & 6.760110 \\
3 & 7.6661 & 8.2854 & 7.6665 & 7.5605 & 7.666142 \\
4 & 6.0772 & 6.2613 & 6.0774 & 5.9913 & 6.077249 \\
5 & 2.9439 & 2.5306 & 2.9438 & 2.9082 & 2.943935 \\
6 & -0.5252 & -1.4920 & -0.5255 & -0.4988 & -0.52517 \\
7 & -3.2463 & -4.5090 & -3.2464 & -3.1680 & -3.24630 \\
8 & -4.5503 & -5.7207 & -4.5505 & -4.4474 & -4.55029 \\
9 & -4.3029 & -5.0009 & -4.3030 & -4.2096 & -4.30286 \\
10 & -2.8484 & -2.8403 & -2.8486 & -2.7939 & -2.84838 \\
\hline
\end{tabular}

Table 4. The resulting values of EI with $h=0.05$ and PMA with $h=0.01$ in some values of $t$ (Example 4).

\begin{tabular}{ccccccccc}
\hline $\boldsymbol{t}$ & $\mathbf{1}$ & $\mathbf{2}$ & $\mathbf{3}$ & $\mathbf{4}$ & $\mathbf{5}$ & $\mathbf{8}$ & $\mathbf{9}$ & $\mathbf{1 0}$ \\
\hline EES & 0.9868 & 1.8907 & 2.4188 & 2.2762 & 1.4744 & -0.3343 & 0.0563 & 0.4257 \\
\hline ETS & 0.9877 & 1.8925 & 2.4199 & 2.2750 & 1.4714 & -0.3322 & 0.0589 & 0.4266 \\
\hline PMA & 0.9955 & 1.8947 & 2.4145 & 2.2635 & 1.4595 & -0.3207 & 0.0700 & 0.4310 \\
\hline
\end{tabular}

particular, the fractional exponential Euler scheme and fractional exponential trapezoidal scheme have been developed to obtain the numerical solution to the Bagley-Torvik equation. The proposed method can be used effectively for the solution of multi-term FDEs so that the Bagley-Torvik equation can serve as one of their prototypes. Implementation issues and error analysis have been treated. The accuracy and validity of the method have been verified by different numerical studies.

\section{References}

1. Oldham, K.B. and Spanier, J., The Fractional Calculus: Theory and Applications of Differentiation and Integration to Arbitrary Order, Academic Press, New York (1974).

2. Podlubny, I., Fractional Differential Equations, Academic Press, San Diego, CA (1999).

3. Metzler, R. and Klafter, J. "The random walks guide to anomalous diffusion: a fractional dynamics approach", Phys. Rep., 339, pp. 1-77 (2000).

4. Rossikhin, Y.A. and Shitikova, M.V. "Application of fractional calculus for dynamic problems of solid mechanics: Novel trends and recent result", Appl. Mech. Rev., 63(1), pp. 010801-52 (2009).

5. Mainardi, F., Fractional Calculus and Waves in Linear Viscoelasticity: An Introduction to Mathematical Models, Imperial College Press, London (2010).

6. Baleanu, D., Diethelm, K., Scalas, E. and Trujillo, J.J., Fractional Calculus: Models and Numerical Methods, 2nd Ed., World Sci. Publishing, Singapore (2016).
7. Atanacković, T.M., Pilipović, S., Stanković, B. and Zorica, D., Fractional Calculus with Applications in Mechanics: Vibrations and Diffusion Processes, WileyISTE, London (2014).

8. Garrappa, R. Mainardi, F. and Maione, G. "Models of dielectric relaxation based on completely monotone functions", Fract. Calc. Appl. Anal., 19(5), pp. 11051160 (2016).

9. Diethelm, K., The Analysis of Fractional Differential Equations, Springer, Berlin (2010).

10. Garrappa, R. and Popolizio, M. "Generalized exponential time differencing methods for fractional order problems", Comput. Math. Appl., 62(3), pp. 876-890 (2011).

11. Garrappa, R. "A family of Adams exponential integrators for fractional linear systems", Comput. Math. Appl., 66(5), pp. 717-727 (2013).

12. Garrappa, R. "Exponential integrators for timefractional partial differential equations", Eur. Phys. J. Special Topics, 222(8), pp. 1913-1925 (2013).

13. Hochbruck, M. and Ostermann, A. "Exponential integrators", Acta Numerica, 19, pp. 209-286 (2010).

14. Higham, N.J., Functions of Matrices: Theory and Computation, SIAM, Philadelphia, PA (2008).

15. Torvik, P.J. and Bagley, R.L. "On the appearance of the fractional derivative in the behavior of real materials", J. Appl. Mech., 51(2), pp. 294-298 (1984).

16. Čermák, J. and Kisela, T. "Exact and discretized stability of the Bagley-Torvik equation", J. Comput. Appl. Math., 269, pp. 53-67 (2014). 
17. Diethelm, K. and Ford, N.J. "Numerical solution of the Bagley-Torvik equation", BIT, 42(3), pp. 490-507 (2002).

18. Mashayekhi, S. and Razzaghi, M. "Numerical solution of the fractional Bagley-Torvik equation by using hybrid functions approximation", Math. Meth. Appl. Sci., 39(3), pp. 353-365 (2016).

19. Mokhtary, P. "Numerical treatment of a well-posed Chebyshev Tau method for Bagley-Torvik equation with high-order of accuracy", Numer. Algorithms, 72(4), pp. 875-891 (2016).

20. Saha Ray, S. "On Haar wavelet operational matrix of general order and its application for the numerical solution of fractional Bagley-Torvik equation", Appl. Math. Comput., 218(9), pp. 5239-5248 (2012).

21. Yüzbaşı, Ş. "Numerical solution of the Bagley-Torvik equation by the Bessel collocation method", Math. Meth. Appl. Sci., 36(3), pp. 300-312 (2013).

22. Luchko, Y. and Gorenflo, R. "An operational method for solving fractional differential equations with the Caputo derivatives", Acta Math. Vietnamica, 24(2), pp. 207-233 (1999).

23. Podlubny, I. "Matrix approach to discrete fractional calculus", Fract. Calc. Appl. Anal., 3(4), pp. 359-386 (2000).

24. Saadatmandi, A. and Dehghan, M. "A new operational matrix for solving fractional-order differential equations", Comput. Math. Appl., 59(3), pp. 1326-1336 (2010).

25. Esmaeili, S., Shamsi, M. and Luchko, Y. "Numerical solution of fractional differential equations with a collocation method based on Müntz polynomials", Comput. Math. Appl., 62(3), pp. 918-929 (2011).

26. Jafari, H., Yousefi, S.A., Firoozjaee, M.A., Momani, S. and Khalique, C.M. "Application of Legendre wavelets for solving fractional differential equations", Comput. Math. Appl., 62, pp. 1038-1045 (2011).

27. Bhrawy, A.H., Taha M.T. and Machado, J.A.T. "A review of operational matrices and spectral techniques for fractional calculus", Nonlinear Dyn., 81(3), pp. 1023-1052 (2015).

28. Jafari, H., Kdkhoda, N., Azadi, M. and Yaghobi, M. "Group classification of the time-fractional KaupKupershmidt equation", Scientia Iranica, 24(1), pp. 302-307 (2017).

29. Diethelm, K. and Ford, N.J. "Analysis of fractional differential equations", J. Math. Anal. Appl., 265(2), pp. 229-248 (2002).
30. Podlubny, I. "Geometric and physical interpretation of fractional integration and fractional differentiation", Fract. Calc. Appl. Anal., 5(4), pp. 367-386 (2002).

31. Esmaeili, S. and Milovanović, G.V. "Nonstandard Gauss-Lobatto quadrature approximation to fractional derivatives", Fract. Calc. Appl. Anal., 17(4), pp. 10751099 (2014).

32. Gorenflo, R., Kilbas, A.A., Mainardi, F. and Rogosin, S.V., Mittag-Leffler Functions, Related Topics and Applications Theory and Applications, Springer, Berlin (2014).

33. Garrappa, R. "Numerical Evaluation of two and three parameter Mittag-Leffler functions", SIAM J. Numer. Anal., 53(3), pp. 1350-1369 (2015).

34. Bagley, R.L. and Calico, R.A. "Fractional order state equations for the control of viscoelastically damped structures", J. Guid. Control Dynam., 14(2), pp. 304311 (1991).

35. Bagley, R.L., Applications of Generalized Derivatives to Viscoelasticity, Air Force Institute of Technology, PhD Dissertation (1979).

36. Lubich, C. "A stability analysis of convolution quadratures for Abel-Volterra integral equations", IMA J. Numer. Anal., 6(1), pp. 87-101 (1986).

37. Lyness, J.N. and Ninham, B.W. "Numerical quadrature and asymptotic expansions", Math. Comp., 21(98), pp. 162-178 (1967).

38. Fitt, A.D., Goodwin, A.R.H., Ronaldson, K.A. and Wakeham, W.A. "A fractional differential equation for a MEMS viscometer used in oil industry", J. Comput. Appl. Math., 229(2), pp. 373-381 (2009).

\section{Biography}

Shahrokh Esmaeili is an Assistant Professor in the Department of Mathematics at University of Kurdistan. He received his BS degree from Amirkabir University of Technology in 1998 and his MSc degree from Tarbiat Modares University in 2000. He started his doctoral program in 2008 and received his $\mathrm{PhD}$ degree from Amirkabir University of Technology in 2011 in Applied Mathematics. His current research interest mainly covers numerical methods for fractional differential equations. 